\title{
The Sarkisov program for Mori fibred Calabi-Yau pairs
}

\author{
Alessio Corti and Anne-Sophie Kaloghiros
}

\begin{abstract}
We prove a version of the Sarkisov program for volume-preserving birational maps of Mori fibred Calabi-Yau pairs valid in all dimensions. Our theorem generalises the theorem of Usnich and Blanc on factorisations of birational maps of $\left(\mathbb{C}^{\times}\right)^{2}$ that preserve the volume form $\frac{d x}{x} \wedge \frac{d y}{y}$.
\end{abstract}

\section{Introduction}

Usnich [Usn06] and Blanc [Bla13] proved that the group of birational automorphisms of $\mathbb{G}_{m}^{2}$ that preserve the volume form $\frac{d x}{x} \wedge \frac{d y}{y}$ is generated by $\mathbb{G}_{m}^{2}, \mathrm{SL}_{2}(\mathbb{Z})$ and the birational map

$$
P:(x, y) \rightarrow\left(y, \frac{1+y}{x}\right) \text {. }
$$

In this paper we prove a generalisation of this result valid in all dimensions. Our theorem generalises the theorem of Usnich and Blanc in the same way that the Sarkisov program [Cor95, HM13] generalises the theorem of Noether and Castelnuovo stating that $\mathrm{Cr}_{2}$ is generated by $\mathrm{PGL}_{3}(\mathbb{C})$ and a standard quadratic transformation

$$
C:\left(x_{0}: x_{1}: x_{2}\right) \rightarrow\left(\frac{1}{x_{0}}: \frac{1}{x_{1}}: \frac{1}{x_{2}}\right) .
$$

Our main result is the following.

Theorem 1.1. A volume-preserving birational map between Mori fibred Calabi-Yau pairs is a composition of volume-preserving Sarkisov links.

It is possible to derive the theorem of Usnich and Blanc from this statement in a similar way that in $[\mathrm{KSC0}, \S 2.5]$ the theorem of Noether-Castelnuovo is derived from the Sarkisov program. This starts from a classification of volume-preserving Sarkisov links and proceeds by assembling batches of Sarkisov links into the map $P$. The resulting proof is completely elementary but long and not relevant to the theme of this paper, which is to prove Theorem 1.1, hence we omit it.

In the rest of the section, we introduce the terminology needed to make sense of the statement and, along the way, we state the more general factorisation theorem (Theorem 1.9) for volume-

Received 20 May 2015, accepted in final form 27 September 2015.

2010 Mathematics Subject Classification 14E30, 14E05, $14 \mathrm{E} 07$.

Keywords: Sarkisov program, Calabi-Yau pairs.

This journal is (c) Foundation Compositio Mathematica 2016. This article is distributed with Open Access under the terms of the Creative Commons Attribution Non-Commercial License, which permits non-commercial reuse, distribution, and reproduction in any medium, provided that the original work is properly cited. For commercial re-use, please contact the Foundation Compositio Mathematica. 


\section{The SARkisov Program FOr Mori FIBred CALABI-YAU PAIRS}

preserving birational maps of general Calabi-Yau pairs. Theorem 1.9 is used in the proof of the main result and is of independent interest. We conclude with some additional remarks.

We refer the reader to [KM98, § 2.3] for the standard definitions of terminal and canonical singularities, and of Kawamata log terminal (klt), divisorial log terminal (dlt) and log canonical (lc) singularities of pairs.

Definition 1.2. (1) Let $X$ be a normal variety and write $F=k(X)$ for its field of fractions. A discrete valuation $\nu: F \rightarrow \mathbb{Z}$ is a geometric valuation with centre on $X$ if

$$
\nu=\text { mult }_{E}, \quad \text { where } E \subset Y \stackrel{f}{\rightarrow} X
$$

is a prime Weil divisor on a normal variety $Y$, and $f: Y \rightarrow X$ is a morphism. We abuse language and identify $E$ with the valuation $\nu=$ mult $_{E}$. The centre of $E$ on $X$ is the scheme-theoretic point $z=f(E) \in X$; we denote it by $\mathrm{z}_{E} X$. We say that $E$ has small centre on $X$ if $z \in X$ is not a divisor, that is, if it has codimension strictly greater than 1 . For all $\mathbb{Q}$-Cartier divisors $D$ on $X$, it makes sense to take the pull-back $f^{\star}(D)$ on $Y$; we write mult $E \bar{D}$ for the coefficient of $E$ in $f^{\star}(D)$.

(2) Let $(X, B)$ be a pair of a normal variety $X$ and a $\mathbb{Q}$-Weil divisor $B \subset X$. The case $B=0$ is allowed. Assume that $K_{X}+B$ is $\mathbb{Q}$-Cartier. Choose a meromorphic differential $\omega \in \Omega_{F / k}^{n}$, where $n=\operatorname{dim} X$. Note that, because $f: Y \rightarrow X$ is birational, $\omega$ is naturally a meromorphic differential on $Y$. In this situation, for all geometric valuations $E$ with centre on $X$ we have

$$
\nu_{E} \omega=a+\operatorname{mult}_{E} \overline{\operatorname{div}_{X} \omega+B}
$$

for some $a \in \mathbb{Q}$. This $a$ depends only on $E$ and the pair $(X, B)$, not on the choice of $\omega$. We call it the discrepancy of $E$ and denote it by $a\left(E, K_{X}+B\right)$.

Definition 1.3. (1) A Calabi-Yau $(C Y)$ pair is a pair $(X, D)$ of a normal variety $X$ and a reduced $\mathbb{Z}$-Weil divisor $D \subset X$ such that $K_{X}+D \sim 0$ is a Cartier divisor linearly equivalent to 0 .

(2) We say that a pair $(X, D)$ has $(t, d l t)$, respectively $(t, l c)$, singularities or that it "is" (t,dlt), respectively (t,lc), if $X$ has terminal singularities and the pair $(X, D)$ has dlt, respectively lc, singularities.

Similarly $(X, D)$ has $(c, d l t)$, respectively $(c, l c)$, singularities or "is" (c,dlt), respectively (c,lc), if $X$ has canonical singularities and the pair $(X, D)$ has dlt, respectively lc, singularities.

(3) We say that a pair $(X, D)$ is $\mathbb{Q}$-factorial if $X$ is $\mathbb{Q}$-factorial.

Remark 1.4. (1) We use the following observation throughout: if $(X, D)$ is a CY pair, then, because $K_{X}+D$ is an integral Cartier divisor, for all geometric valuations $E$, we have $a\left(E, K_{X}+\right.$ $D) \in \mathbb{Z}$. If in addition $(X, D)$ is lc or dlt, then $a\left(E, K_{X}+D\right) \leqslant 0$ implies $a(E, K+D)=-1$ or 0 .

(2) If $(X, D)$ is a dlt CY pair, then automatically it is (c,dlt). More precisely if $E$ is a geometric valuation with small centre on $X$ and if the centre $\mathrm{z}_{X} E$ is an element of $\operatorname{Supp} D$, then $a\left(E, K_{X}\right)>0$.

Indeed, consider a valuation $E$ with small centre on $X$. Then

$$
a\left(E, K_{X}\right)=a\left(E, K_{X}+D\right)+\operatorname{mult}_{E} \bar{D}
$$

therefore $a\left(E, K_{X}\right) \leqslant 0$ implies $a\left(E, K_{X}+D\right) \leqslant 0$ and then, because $K_{X}+D$ is a Cartier divisor, either $a\left(E, K_{X}+D\right)=-1$, which is impossible because by definition of dlt, see Remark 2.4 below, $z=\mathrm{z}_{X} E \in X$ is smooth, or $a\left(E, K_{X}+D\right)=0$ and $\operatorname{mult}_{E} \bar{D}=0$. 


\section{A. Corti And A.-S. Kaloghiros}

Definition 1.5. A $\log$ resolution of a pair $(X, B)$ is a projective morphism $f: Y \rightarrow X$ such that

(i) $Y$ is smooth, the exceptional set $\operatorname{Ex} f$ is of pure codimension 1;

(ii) for $B^{\prime}$ the proper transform of $B$, the union of $\operatorname{Ex} f \cup \operatorname{Supp} B^{\prime} \subset Y$ is a simple normal crossing (snc) divisor.

Definition 1.6. Let $\left(X, D_{X}\right)$ and $\left(Y, D_{Y}\right)$ be CY pairs. A birational map $\varphi: X \rightarrow Y$ is volume preserving if for all geometric valuations $E$ with centre on both $X$ and $Y$, we have $a\left(E, K_{X}+\right.$ $\left.D_{X}\right)=a\left(E, K_{Y}+D_{Y}\right)$.

This is equivalent to saying that there exists a common log resolution

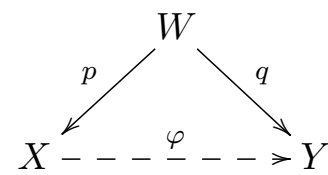

such that $p^{\star}\left(K_{X}+D_{X}\right)=q^{\star}\left(K_{Y}+D_{Y}\right){ }^{1}$

Remark 1.7. If $\left(X, D_{X}\right)$ is a CY pair and $X$ is proper, then there is a unique (up to multiplication by a nonzero constant) rational differential $\omega_{X} \in \Omega_{k(X) / k}^{n}$ such that $D_{X}+\operatorname{div}_{X} \omega_{X} \geqslant 0$. Similarly, if $Y$ is also proper, there is a distinguished rational differential $\omega_{Y}$ on $Y$. To say that $\varphi$ is volume preserving is to say $\varphi_{\star} \omega_{X}=\omega_{Y}$.

Volume-preserving maps are called crepant birational in [Kol13].

Remark 1.8. It is obvious from the definition that the composition of two volume-preserving maps between proper varieties is volume preserving.

The first step in the proof of Theorem 1.1 is the following general factorisation theorem for volume-preserving birational maps between lc CY pairs, which is of independent interest. See [KX15, Lemma 12(4)] for a similar statement.

Theorem 1.9. Let $(X, D)$ and $\left(X^{\prime}, D^{\prime}\right)$ be lc $C Y$ pairs, and let $\varphi: X \rightarrow X^{\prime}$ be a volumepreserving birational map. Then there are $\mathbb{Q}$-factorial $(t, d l t) C Y$ pairs $\left(Y, D_{Y}\right),\left(Y^{\prime}, D_{Y^{\prime}}\right)$ and a commutative diagram of birational maps

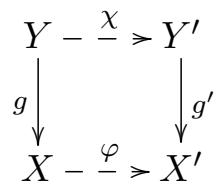

where

(i) the morphisms $g: Y \rightarrow X, g^{\prime}: Y^{\prime} \rightarrow X^{\prime}$ are volume preserving;

(ii) the morphism $\chi: Y \rightarrow Y^{\prime}$ is a volume-preserving isomorphism in codimension 1 which is a composition of volume-preserving Mori flips, flops and inverse flips (not necessarily in that order).

Definition 1.10. A Mori fibred $(M f) C Y$ pair is a $\mathbb{Q}$-factorial $(\mathrm{t}, \mathrm{lc}) \mathrm{CY}$ pair $(X, D)$ together with a Mori fibration $f: X \rightarrow S$. Recall that this means that $f_{\star} \mathcal{O}_{X}=\mathcal{O}_{S}$, the divisor $-K_{X}$ is $f$-ample, and $\rho(X)-\rho(S)=1$.

${ }^{1}$ By this we mean that for all meromorphic differentials $\omega \in \Omega_{k(X) / k}^{n}$, we have $p^{\star}\left(D_{X}+\operatorname{div}_{X} \omega\right)=q^{\star}\left(D_{Y}+\right.$ $\left.\operatorname{div}_{Y} \varphi_{*} \omega\right)$. 


\section{The SARKisov Program for Mori fibred CAlabi-Yau PAIRS}

Terminology 1.11. We use the following terminology throughout.

(1) A Mori divisorial contraction is an extremal divisorial contraction $f: Z \rightarrow X$ from a $\mathbb{Q}$-factorial terminal variety $Z$ of an extremal ray $R$ with $K_{Z} \cdot R<0$. In particular, $X$ also has $\mathbb{Q}$-factorial terminal singularities.

If $\left(Z, D_{Z}\right)$ and $\left(X, D_{X}\right)$ are (t,lc) CY pairs, then it makes sense to say that $f$ is volume preserving. In this context, this is equivalent to saying that $K_{Z}+D_{Z}=f^{\star}\left(K_{X}+D_{X}\right)$ and, in particular, $D_{X}=f_{\star}\left(D_{Z}\right)$.

A birational map $t: Z \rightarrow Z^{\prime}$ is a Mori flip if $Z$ has $\mathbb{Q}$-factorial terminal singularities and $t$ is the flip of an extremal ray $R$ with $K_{Z} \cdot R<0$. Note that this implies that $Z^{\prime}$ has $\mathbb{Q}$-factorial terminal singularities.

An inverse Mori flip is the inverse of a Mori flip.

(2) A birational map $t: Z \rightarrow Z^{\prime}$ is a Mori flop if $Z$ and $Z^{\prime}$ have $\mathbb{Q}$-factorial terminal singularities and $t$ is the flop of an extremal ray $R$ with $K_{Z} \cdot R=0$.

Again, if $\left(Z, D_{Z}\right)$ and $\left(Z^{\prime}, D_{Z^{\prime}}\right)$ are (t,lc) CY pairs, it makes sense to say that $t$ is volume preserving. One can see that this just means that $D_{Z^{\prime}}=t_{\star} D_{Z}$.

Definition 1.12. Let $(X, D)$ and $\left(X^{\prime}, D^{\prime}\right)$ be Mf CY pairs with Mori fibrations $X \rightarrow S$ and $X^{\prime} \rightarrow S^{\prime}$. A volume-preserving Sarkisov link is a volume-preserving birational map $\varphi: X \rightarrow X^{\prime}$ that is a Sarkisov link in the sense of [Cor95]. Thus $\varphi$ is of one of the following types:

(I) A link of type $I$ is a commutative diagram

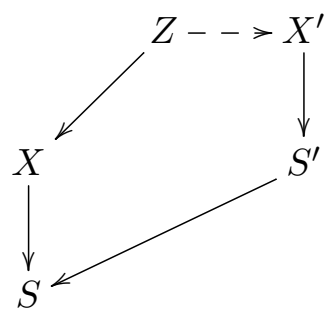

where $Z \rightarrow X$ is a Mori divisorial contraction and $Z \rightarrow X^{\prime}$ is a sequence of Mori flips, flops and inverse flips.

(II) A link of type II is a commutative diagram

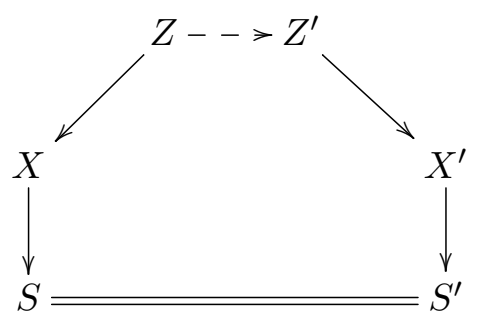

where $Z \rightarrow X$ and $X^{\prime} \rightarrow Z^{\prime}$ are Mori divisorial contractions and $Z \rightarrow Z^{\prime}$ is a sequence of Mori flips, flops and inverse flips.

(III) A link of type III is the inverse of a link of type I. 


\section{A. Corti And A.-S. Kaloghiros}

(IV) A link of type $I V$ is a commutative diagram

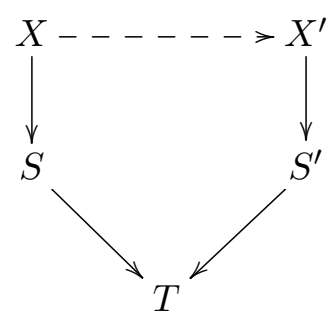

where $X \rightarrow X^{\prime}$ is a sequence of Mori flips, flops and inverse flips.

Remark 1.13. It follows from the definition of Sarkisov link that all the divisorial contractions, flips, etc. that constitute it are volume preserving; in particular, all varieties in sight are naturally and automatically $(\mathrm{t}, \mathrm{lc}) \mathrm{CY}$ pairs.

In order to appreciate the statement of our main theorem, Theorem 1.1, it is important to be aware that, although all Mf CY pairs are only required to have lc singularities as pairs, we insist that all varieties in sight have $\mathbb{Q}$-factorial terminal singularities. Our factorisation theorem is at the same time a limiting case of the Sarkisov program for pairs [BM97] and a Sarkisov program for varieties [Cor95, HM13]. The Sarkisov program for pairs usually spoils the singularities of the underlying varieties, while the Sarkisov program for varieties does not preserve singularities of pairs. The proof our main result is a balancing act between singularities of pairs and of varieties.

We expect that it will be possible in some cases to classify all volume-preserving Sarkisov links and hence give useful presentations of groups of volume-preserving birational maps of interesting Mf CY pairs. We plan to return to these questions in the near future.

The paper is structured as follows. In Section 2 we develop some general results on CY pairs and volume-preserving maps between them and prove Theorem 1.9; in Section 3 we prove Theorem 1.1.

\section{Birational geometry of CY pairs}

Definition 2.1. Let $(X, D)$ be a lc CY pair, and let $f: W \rightarrow X$ be a birational morphism. The log transform of $D$ is the divisor

$$
D_{W}=f^{b}(D)=\sum_{a\left(E, K_{X}+D\right)=-1} E,
$$

where the sum is over all prime divisors $E \subset W$. (Note that $D_{W}$ contains the proper transform of $D$.)

Lemma 2.5 is a refinement of $\left[\mathrm{Kol}^{+} 92\right.$, Theorem 17.10] and [Fuj11, Theorem 4.1]. In order to state it we need a definition.

DeFinition 2.2. Let $X$ be a normal variety. A geometric valuation with centre on $X$ is a valuation of the function field $K(X)$ of the form mult $_{E}$, where $E \subset Y$ is a divisor on a normal variety $Y$ with a birational morphism $f: Y \rightarrow X$. The centre of $E$ on $X$, denoted $\mathrm{z}_{X} E$, is the generic point of $f(E)$.

Let $(X, D)$ be a lc pair. The non-klt set is the set

$$
\operatorname{NKLT}(X, D)=\left\{z \in X \mid z=\mathrm{z}_{X} E, \text { where } a\left(E, K_{X}+D\right)=-1\right\},
$$




\section{The SARkisov Program FOr Mori FIBred CALABI-YAU PAIRS}

where $E$ is a geometric valuation of the function field of $X$ with centre the scheme-theoretic point $\mathrm{z}_{X} E \in X$.

Warning 2.3. Our notion of non-klt set departs from common usage. Most authors work with the non-klt locus - the Zariski closure of our non-klt set - which they denote $\operatorname{nklt}(X, D)$ (in lower case letters).

Remark 2.4. We use the following statement throughout: it is part of the definition of dlt pairs [KM98, Definition 2.37] that if $(X, D)$ is dlt, where $D=\sum_{i=1}^{r} D_{i}$ with $D_{i} \subset X$ a prime divisor, then $\operatorname{NKLT}(X, D)$ is the set of generic points of the

$$
D_{I}=\cap_{i \in I} D_{i}, \quad \text { where } I \subset\{1, \ldots, r\}
$$

and $X$ is nonsingular at all these points.

Lemma 2.5. Let $(X, D)$ be a lc $C Y$ pair where $X$ is not necessarily proper, let $f: W \rightarrow X$ be a $\log$ resolution, and let $D_{W}=f^{b}(D)$.

The minimal model program $(M M P)$ for $K_{W}+D_{W}$ over $X$ with scaling of a divisor ample over $X$ exists and terminates at a minimal model $\left(Y, D_{Y}\right)$ over $X$ (that is, $K_{Y}+D_{Y}$ is nef over $X)$. More precisely, this MMP consists of a sequence of steps

$$
\left(W, D_{W}\right)=\left(W_{0}, D_{0}\right) \stackrel{t_{0}}{\rightarrow} \cdots\left(W_{i}, D_{i}\right)^{\stackrel{t_{i}}{\rightarrow}}\left(W_{i+1}, D_{i+1}\right) \cdots \rightarrow\left(W_{N}, D_{N}\right)=\left(Y, D_{Y}\right),
$$

where $t_{i}: W_{i} \rightarrow W_{i+1}$ is the divisorial contraction or flip of an extremal ray $R_{i} \subset \overline{\mathrm{NE}}\left(W_{i} / X\right)$ with $\left(K_{W_{i}}+D_{i}\right) \cdot R_{i}<0$. We denote by $g_{i}: W_{i} \rightarrow X$ the structure morphism and by $g:\left(Y, D_{Y}\right) \rightarrow$ $(X, D)$ the end result.

(i) For all $i$, denote by $h_{i}: W \rightarrow W_{i}$ the induced map. For all $i$, there are Zariski-open neighbourhoods

$$
\operatorname{NKLT}\left(W, D_{W}\right) \subset U \text { and } \operatorname{NKLT}\left(W_{i}, D_{i}\right) \subset U_{i}
$$

such that $h_{i} \mid U: U \rightarrow U_{i}$ is an isomorphism.

(ii) We have $D_{Y}=g^{b} D$ and $K_{Y}+D_{Y}=g^{\star}\left(K_{X}+D\right)$ (that is, $g$ is a dlt crepant blow-up).

(iii) The pair $\left(Y, D_{Y}\right)$ is a $(t, d l t) C Y$ pair. In particular, $Y$ has terminal singularities.

(iv) The map $h: W \rightarrow Y$ contracts precisely the prime divisors $E \subset W$ with $a\left(E, K_{X}+D\right)>0$. In other words, an $f$-exceptional divisor $E \subset W$ is not contracted by the map $h: W \rightarrow Y$ if and only if $a\left(E, K_{X}+D\right)=0$ or -1 .

Proof. The MMP exists by [Fuj11, Theorem 4.1]. In the rest of the proof we use the following well-known fact: if $E$ is a geometric valuation with centre on $W$, then for all $i$

$$
a\left(E, K_{W_{i}}+D_{i}\right) \leqslant a\left(E, K_{W_{i+1}}+D_{i+1}\right),
$$

and the inequality is an equality if and only if $t_{i}: W_{i} \rightarrow W_{i+1}$ is an isomorphism in a neighbourhood of $z_{i}=\mathrm{z}_{W_{i}} E$. In particular, this at once implies $N K L T\left(W_{i}, D_{i}\right) \supset \operatorname{NKLT}\left(W_{i+1}, D_{i+1}\right)$, and the two sets are equal if and only if there exist Zariski-open subsets as in assertion (i), if and only if for all $E$ with $a\left(E, K+D_{i}\right)=-1$ the morphism $t_{i}$ is an isomorphism in a neighbourhood of $z_{W_{i}} E$.

Now write

$$
K_{W}+D_{W}=f^{\star}\left(K_{X}+D\right)+F
$$

with $F$ a strictly effective $f$-exceptional divisor having no component in common with $D_{W}$. We are running an $F$-MMP, hence if $F_{i} \subset W_{i}$ denotes the image of $F$, then the exceptional set of 


\section{A. Corti and A.-S. Kaloghiros}

the map $t_{i}: W_{i} \rightarrow W_{i+1}$ is contained in $\operatorname{Supp} F_{i}$; see [Kol13, $\left.\S 1.35\right]$. From this it follows that $h_{i}$ is an isomorphism from $W \backslash \operatorname{Supp} F$ to its image in $W_{i}$. At the start, $D_{W}$ has no components in common with $F$ and $\operatorname{Supp}\left(D_{W} \cup F\right)$ is a snc divisor; thus, if $a\left(E, K_{W}+D_{W}\right)=-1$, then $z_{W} E \notin F$. It follows that $\operatorname{NKLT}\left(W, D_{W}\right) \subset \operatorname{NKLT}\left(W_{i}, D_{i}\right)$. Together with what we said, this implies assertion (i).

For assertion (ii), it is obvious that for all $i$, we have $D_{i}=g_{i}^{b} D$. By the negativity lemma [KM98, Lemma 3.39] $F_{i} \neq 0$ implies $F_{i}$ not nef, so the MMP ends at $g_{N}=g: W_{N}=Y \rightarrow X$ when $F_{N}=0$; that is, $K_{Y}+D_{Y}=g^{\star}\left(K_{X}+D\right)$.

For assertion (iii) we need to show that $Y$ has terminal singularities. Suppose that $E$ is a valuation with small centre $z_{Y} E$ on $Y$. By Remark 1.4, either $a\left(E, K_{X}\right)>0$ or

$$
a\left(E, K_{X}\right)=a\left(E, K_{X}+D\right)=0 \quad \text { and } \quad \mathrm{z}_{Y} E \notin \operatorname{Supp} D_{Y},
$$

and we show that this second possibility leads to a contradiction. Write $z_{i}=\mathrm{z}_{W_{i}} E$. Note that $z_{i} \in W_{i}$ is never a divisor, for this would imply $a\left(E, K_{Y}\right)>0$. By what we said at the start of the proof, for all $i$, we have $a\left(E, K_{W_{i}}+D_{i}\right) \leqslant a\left(E, K_{W_{i+1}}+D_{i+1}\right)$, with strict inequality if and only if $t_{i}: W_{i} \rightarrow W_{i+1}$ is not an isomorphism in a neighbourhood of $z_{i} \in W_{i}$. There must be a point where strict inequality occurs, otherwise $z_{0} \notin D_{0}$ and $W=W_{0}$ is not terminal in a neighbourhood of $z_{0}$. This, however, implies $a\left(E, K_{W}+D\right)<0$, that is, $a\left(E, K_{W}+D_{W}\right)=-1$ and then by assertion (i), the morphism $h: W \rightarrow Y$ is an isomorphism in a neighbourhood of $z_{0}$, again a contradiction.

Statement (iv) is obvious.

Example 2.6. This example should help appreciate the statement of Theorem 1.9 and the subtleties of its proof. Let $\pi: W \rightarrow \mathbb{P}^{1} \times \mathbb{P}^{1}$ be the total space of the vector bundle $\mathcal{O}(-1,-2)$ on $\mathbb{P}^{1} \times \mathbb{P}^{1}$, let $E \subset W$ be the zero section, and let $D_{W}=\pi^{\star} p_{1}^{\star}(0)$, where $p_{1}: \mathbb{P}^{1} \times \mathbb{P}^{1} \rightarrow \mathbb{P}^{1}$ is the projection to the first factor. Note that $D_{W} \cap E$ is a ruling in $E$ and a -2-curve in $D_{W}$. Let $f: W \rightarrow Y$ be the contraction of $E$ along the first ruling, and let $f^{\prime}: W \rightarrow Y^{\prime}$ be the contraction along the second ruling. Then $(Y, D)$ and $\left(Y^{\prime}, D^{\prime}\right)$ are both dlt, $Y^{\prime}$ is terminal, $Y$ is canonical but not terminal, and the map $Y \rightarrow Y^{\prime}$ is volume preserving.

Proof of Theorem 1.9. Let

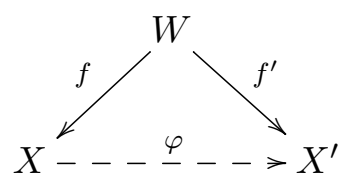

be a common $\log$ resolution. Since $\varphi$ is volume preserving, for all geometric valuations $E$ we have $a\left(E, K_{X}+D\right)=a\left(E, K_{X^{\prime}}+D^{\prime}\right)$ and

$$
K_{W}+D_{W}=f^{\star}\left(K_{X}+D\right)+F=f^{\prime \star}\left(K_{X^{\prime}}+D^{\prime}\right)+F,
$$

where $D_{W}=f^{b} D=f^{\prime b} D^{\prime}$ and

$$
F=\sum_{a_{E}\left(K_{X}+D\right)>0} a\left(E, K_{X}+D\right) E=\sum_{a_{E}\left(K_{X^{\prime}}+D^{\prime}\right)>0} a\left(E, K_{X^{\prime}}+D^{\prime}\right) E .
$$

Let $g:\left(Y, D_{Y}\right) \rightarrow(X, D)$ and $g^{\prime}:\left(Y^{\prime}, D_{Y^{\prime}}\right) \rightarrow\left(X^{\prime}, D^{\prime}\right)$ be the end products of the $\left(K_{W}+D_{W}\right)$ MMP over $X$ and $X^{\prime}$ as in Lemma 2.5, and denote by $\chi: Y \rightarrow Y^{\prime}$ the induced map. By Lemma 2.5(iv), this map $\chi$ is an isomorphism in codimension 1.

Denote by $t: W \rightarrow Y$ and $t^{\prime}: W \rightarrow Y^{\prime}$ the obvious maps and write $\operatorname{NKLT}\left(W, D_{W}\right) \subset$ $U_{W}=W \backslash \operatorname{Supp} F$; by Lemma 2.5(i), the restrictions $t \mid U_{W}$ and $t^{\prime} \mid U_{W}$ are isomorphisms onto 


\section{The SARKisov Program for Mori fibred CAlabi-Yau PAIRS}

their images $\operatorname{NKLT}\left(Y, D_{Y}\right) \subset U \subset Y$ and $\operatorname{NKLT}\left(Y^{\prime}, D_{Y^{\prime}}\right) \subset U^{\prime} \subset Y^{\prime}$. It follows from this that $\chi \mid U$ maps $U$ isomorphically to $U^{\prime}$.

In the rest of the proof if $N$ is a divisor on $Y$ we denote by $N^{\prime}$ its transform on $Y^{\prime}$ and conversely; because $\chi$ is an isomorphism in codimension 1, it is clear what the notation means.

Let us choose, as we can by what we just said, an ample $\mathbb{Q}$-divisor $L^{\prime}$ on $Y^{\prime}$ general enough that both $\left(Y^{\prime}, D_{Y^{\prime}}+L^{\prime}\right)$ and $\left(Y, D_{Y}+L\right)$ are dlt. Let $0<\varepsilon \ll 1$ be small enough that $A^{\prime}=L^{\prime}-\varepsilon D^{\prime}$ is ample. Note that, again by what we said above, if we set $\Theta^{\prime}=L^{\prime}+(1-\varepsilon) D_{Y^{\prime}}$, both pairs $\left(Y^{\prime}, \Theta^{\prime}\right)$ and $(Y, \Theta)$ are klt.

Since $K_{Y^{\prime}}+\Theta^{\prime} \sim_{\mathbb{Q}} A^{\prime}$ is ample, $\left(Y^{\prime}, \Theta_{Y^{\prime}}\right)$ is the log canonical model of $(Y, \Theta)$. It follows that $\chi$ is the composition of finitely many [BCHM10, Corollary 1.4.2] flips

$$
\chi: Y=Y_{0} \stackrel{\chi_{0}}{\rightarrow} Y_{1} \stackrel{\chi_{1}}{\rightarrow} \ldots \stackrel{\chi_{N-1}}{\rightarrow--\rightarrow} Y_{N}=Y^{\prime}
$$

of the MMP for $K_{Y}+\Theta$. If $N$ is a divisor on $Y$, denote by $N_{i}$ its transform on $Y_{i}$. For all $i$, the map $\chi_{i}$ is a $\left(K_{Y_{i}}+\Theta_{i}\right)$-flip and, at the same time, a $\left(K_{Y_{i}}+D_{i}\right)$-flop, and hence all pairs $\left(Y_{i}, D_{i}\right)$ are lc. We next argue that all $\left(Y_{i}, D_{i}\right)$ are in fact (t,dlt).

Because the MMP is a MMP for $A \sim_{\mathbb{Q}} K_{Y}+\Theta$, the exceptional set of $\chi_{i}$ is contained in $\operatorname{Supp} A_{i}$. From this it follows that for $U_{0}=U$, the restriction $\chi_{0} \mid U_{0}$ is an isomorphism onto its image, which we denote by $U_{1}$ and, by induction on $i$, the restriction $\chi_{i} \mid U_{i}$ is an isomorphism onto its image, which we denote by $U_{i+1}$. We show by induction that for all $i$, the set $U_{i}$ is a Zariski neighbourhood of $\operatorname{NKLT}\left(Y_{i}, D_{i}\right)$, so that $\chi_{i}$ is a local isomorphism at the generic point of each $z \in \operatorname{NKLT}\left(Y_{i}, D_{i}\right)$ and $\left(Y_{i}, D_{i}\right)$ is a dlt pair. Indeed, assuming the statement for $i<k$, consider $\chi_{k}: Y_{k} \rightarrow Y_{k+1}$. Let $E$ be a valuation with discrepancy $a\left(E, K_{Y_{k+1}}+D_{k+1}\right)=-1$; then also $a\left(E, K_{Y_{k}}+D_{k}\right)=-1$, thus $z_{k}=\mathrm{z}_{Y_{k}} E \in \operatorname{NKLT}\left(Y_{k}, D_{k}\right) \subset U_{k}$ and then by what we just said $\chi_{k}$ is an isomorphism at $z_{k}$, hence $z_{k+1}=\chi_{k}\left(z_{k}\right) \in U_{k+1}$. This shows that all $\left(Y_{i}, D_{i}\right)$ are dlt.

Finally, we prove that for all $i$, the variety $Y_{i}$ is terminal. Assume for a contradiction that $Y_{j}$ is not terminal. By Remark 1.4(2), the variety $Y_{j}$ is canonical and there is a geometric valuation $E$ with $a\left(E, K_{Y_{j}}\right)=a\left(E, K_{Y_{j}}+D_{j}\right)=\operatorname{mult}_{E} \bar{D}_{j}=0$, and then also $a\left(E, K_{Y}+D_{Y}\right)=$ $a\left(E, K_{Y^{\prime}}+D_{Y^{\prime}}\right)=0$. Since $Y$ is terminal, $a\left(E, K_{Y}\right)>0$, and $\mathrm{z}_{Y} E \notin U$ and $\mathrm{z}_{W} E \in \operatorname{Supp} F$, but then $a\left(E, K_{Y}+D_{Y}\right)>a\left(E, K_{W}+D_{W}\right)$, so that we must have $a\left(E, K_{W}+D_{W}\right)=-1$, that is, $\mathrm{z}_{W} E \in \operatorname{NKLT}\left(W, D_{W}\right) \subset U_{W}$, which gives a contradiction.

\section{Sarkisov program under $Y$}

\subsection{Basic setup}

We fix the following situation, which we keep in force throughout this section:

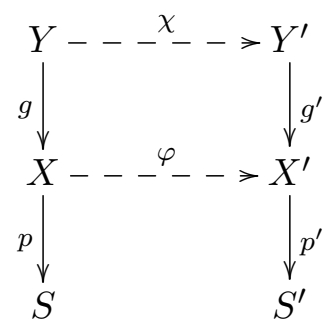

where

(i) $Y$ and $Y^{\prime}$ have $\mathbb{Q}$-factorial terminal singularities and $g: Y \rightarrow X$ and $g^{\prime}: Y \rightarrow X^{\prime}$ are 


\section{A. Corti And A.-S. Kaloghiros}

birational morphisms;

(ii) $\chi: Y \rightarrow Y^{\prime}$ is the composition of Mori flips, flops and inverse flips;

(iii) $p: X \rightarrow S$ and $p^{\prime}: X^{\prime} \rightarrow S^{\prime}$ are Mfs.

The goal of this section is to prove Theorem 3.3 below. In the final short Section 4 we show that Theorems 1.9 and 3.3 imply Theorem 1.1. The proof of Theorem 3.3 is a variation on the proof of [HM13].

Definition 3.1. A birational map $f: X \rightarrow Y$ is contracting if $f^{-1}$ contracts no divisors.

Remark 3.2. If a birational map $f: X \rightarrow Y$ is contracting, then it makes sense to pull back $\mathbb{Q}$-Cartier (R-Cartier) divisors from $Y$ to $X$. Choose a normal variety $W$ and a factorisation

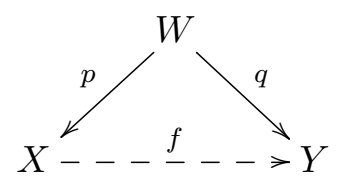

with $p$ and $q$ proper birational morphisms. If $D$ is a $\mathbb{Q}$-Cartier $\mathbb{Q}$-divisor or an $\mathbb{R}$-Cartier $\mathbb{R}$-divisor on $Y$, the pull-back $f^{\star}(D)$ is defined as

$$
f^{\star}(D)=p_{\star} q^{\star}(D)
$$

(this is easily seen to be independent of the factorisation).

Theorem 3.3. The birational map $\varphi: X \rightarrow X^{\prime}$ is a composition of links $\varphi_{i}: X_{i} / S_{i} \rightarrow-$ $X_{i+1} / S_{i+1}$ of the Sarkisov program, where all the maps $Y \rightarrow X_{i}$ are contracting.

Terminology 3.4. We say that the link $\varphi_{i}: X_{i} / S_{i} \rightarrow X_{i+1} / S_{i+1}$ is under $Y$ if the maps $Y \rightarrow X_{i}$ and $Y \rightarrow X_{i+1}$ are contracting.

\subsection{Finitely-generated divisorial rings}

\subsubsection{General theory}

Definition 3.5. Let $f: X \rightarrow Y$ be a contracting birational map.

Let $D_{X}$ be an $\mathbb{R}$-divisor. We say that $f$ is $D_{X}$-nonpositive (respectively, $D_{X}$-negative) if $D_{Y}=f_{\star} D_{X}$ is $\mathbb{R}$-Cartier and

$$
D_{X}=f^{\star}\left(D_{Y}\right)+\sum_{\mathrm{E}} \sum_{f \text {-exceptional }} a_{E} E,
$$

where $a_{E} \geqslant 0$ (respectively, $a_{E}>0$ ) for all $E$.

Note the special case $D_{X}=K_{X}$ in this definition.

Definition 3.6. Let $X / Z$ be a normal variety, proper over $Z$, and let $D$ be an $\mathbb{R}$-divisor on $X$.

(1) A semiample model of $D$ is a $D$-nonpositive contracting birational map $\varphi: X \rightarrow Y$ to a normal variety $Y / Z$, proper over $Z$, such that $D_{Y}=\varphi_{\star} D$ is semiample over $Z$.

(2) An ample model of $D$ is a rational map $h: X \rightarrow W$ to a normal variety $W / Z$, projective over $Z$, together with an ample $\mathbb{R}$-Cartier divisor $A$, such that there is a factorisation $h=g \circ f$ :

$$
X \stackrel{f}{\rightarrow} Y \stackrel{g}{\rightarrow} W,
$$

where $f: X \rightarrow Y$ is a semiample model of $D$, the map $g: Y \rightarrow W$ is a morphism, and $D_{Y}=$ $g^{\star}(A)$. 


\section{The SARkisov program for Mori fibred CALABI-YAU PAIRS}

Remark 3.7. Let $X / Z$ be a normal variety, proper over $Z$, and let $D$ be an $\mathbb{R}$-divisor on $X$.

(1) Suppose that $W / Z$ is normal, let $A$ be an ample $\mathbb{R}$-divisor on $W$, and let $h: X \rightarrow W$ be an ample model of $D$. If $f: X \rightarrow Y$ is a semiample model of $D$, then the induced rational map $g: Y \rightarrow W$ is a morphism and $D_{Y}=g^{\star} A$.

(2) All ample models of $D$ are isomorphic over $Z$.

We refer to $[\mathrm{KKL} 12, \S 3]$ for basic terminology on divisorial rings.

Theorem 3.8 ([KKL12, Theorem 4.2]). Let $X$ be a projective $\mathbb{Q}$-factorial variety, and let $\mathcal{C} \subseteq$ $\operatorname{Div}_{\mathbb{R}}(X)$ be a rational polyhedral cone containing a big divisor ${ }^{2}$ such that the ring $\mathfrak{R}=R(X, \mathcal{C})$ is finitely generated. Then there exist a finite rational polyhedral fan $\Sigma$ and a decomposition

$$
\text { Supp } \mathfrak{R}=|\Sigma|=\coprod_{\sigma \in \Sigma} \sigma
$$

such that we have the following:

(i) For all $\sigma \in \Sigma$ there exist a normal projective variety $X_{\sigma}$ and a rational map $\varphi_{\sigma}: X \rightarrow X_{\sigma}$ such that for all $D \in \sigma$, the map $\varphi_{\sigma}$ is the ample model of $D$. If $\sigma$ contains a big divisor, then for all $D \in \bar{\sigma}$, the map $\varphi_{\sigma}$ is a semiample model of $D$.

(ii) For all $\tau \subseteq \bar{\sigma}$ there exists a morphism $\varphi_{\sigma \tau}: X_{\sigma} \longrightarrow X_{\tau}$ such that the diagram

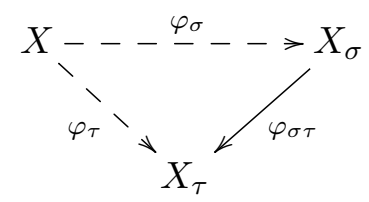

commutes.

Remark 3.9. (1) Under the assumptions of Theorem 3.8, if a cone $\sigma \in \Sigma$ intersects the interior of Supp $\mathfrak{R}$, then it consists of big divisors (this is because the big cone is the interior of the pseudoeffective cone). This holds in particular if $\sigma$ is of maximal dimension.

(2) Theorem 3.8(ii) follows immediately from part (i) and Remark 3.7(1).

Definition 3.10. Let $X$ be a projective $\mathbb{Q}$-factorial variety, and let $\mathcal{C} \subseteq \operatorname{Div}_{\mathbb{R}}(X)$ be a rational polyhedral cone containing a big divisor such that the ring $\mathfrak{R}=R(X, \mathcal{C})$ is finitely generated. We say that $\mathcal{C}$ is generic if

(i) for all $\sigma \in \Sigma$ of maximal dimension (that is, $\operatorname{dim} \sigma=\operatorname{dim} \operatorname{Supp} \Re$ ), the variety $X_{\sigma}$ is $\mathbb{Q}$-factorial;

(ii) for all $\sigma \in \Sigma$, not necessarily of maximal dimension, and all $\tau \subset \bar{\sigma}$ of codimension 1 , the morphism $X_{\sigma} \rightarrow X_{\tau}$ has relative Picard rank $\rho\left(X_{\sigma} / X_{\tau}\right) \leqslant 1$.

Notation 3.11. If $V$ is a $\mathbb{R}$-vector space and $v_{1}, \ldots, v_{k} \in V$, then we denote by

$$
\left\langle v_{1}, \ldots, v_{k}\right\rangle=\sum_{i=1}^{k} \mathbb{R}_{\geqslant 0} v_{i}
$$

the convex cone in $V$ spanned by the $v_{i}$.

\footnotetext{
${ }^{2}$ We need to assume that $\mathcal{C}$ contains a big divisor so we can say: if $D \in \mathcal{C}$ is pseudo effective, then $D$ is effective.
} 


\section{A. Corti And A.-S. Kaloghiros}

Lemma 3.12. Let $X$ be a projective $\mathbb{Q}$-factorial variety, and let $\mathcal{C} \subseteq \operatorname{Div}_{\mathbb{R}}(X)$ be a generic rational polyhedral cone containing a big divisor.

Let $D_{1}, \ldots, D_{k} \in \mathcal{C}$ be such that the cone $\left\langle D_{1}, \ldots, D_{k}\right\rangle$ contains a big divisor, and let $\varepsilon>0$. There exist $D_{1}^{\prime}, \ldots, D_{k}^{\prime} \in \mathcal{C}$ with $\left\|D_{i}-D_{i}^{\prime}\right\|<\varepsilon$ such that the cone $\left\langle D_{1}^{\prime}, \ldots, D_{k}^{\prime}\right\rangle$ is generic.

Proof. Make sure that all cones $\left\langle D_{i_{1}}^{\prime}, \ldots, D_{i_{c}}^{\prime}\right\rangle$ for $i_{1}, \ldots, i_{c} \in\{1, \ldots, k\}$ intersect all cones $\sigma \in \Sigma$ properly.

Theorem 3.13. Let $X$ be a projective $\mathbb{Q}$-factorial variety, let $\Delta_{1}, \ldots, \Delta_{r} \geqslant 0$ big $\mathbb{Q}$-divisors on $X$ such that all pairs $\left(X, \Delta_{i}\right)$ are klt, and let

$$
\mathcal{C}=\left\langle K_{X}+\Delta_{1}, \ldots, K_{X}+\Delta_{r}\right\rangle .
$$

Then $\mathfrak{R}=R(X, \mathcal{C})$ is finitely generated, and if $\operatorname{Supp} \mathfrak{R}$ spans $N_{\mathbb{R}}^{1}(X)$ as a vector space, then $\mathcal{C}$ is generic.

For the proof see for example [KKL12, Theorem 4.5]. Note that the assumptions readily imply that Supp $\mathfrak{R}$ contains big divisors. The finite generation of $\mathfrak{R}$ is of course the big theorem of [BCHM10].

SETUP 3.14. In what follows we work with a pair $\left(X, G_{X}\right)$, where $X$ is $\mathbb{Q}$-factorial and

(i) $G_{X}$ is a $\mathbb{Q}$-linear combination of irreducible mobile ${ }^{3}$ divisors;

(ii) $\left(X, G_{X}\right)$ is terminal;

(iii) $K_{X}+G_{X}$ is not pseudoeffective.

Assumption (i) implies that when we run the MMP for $K_{X}+G_{X}$, no component of $G_{X}$ is ever contracted, so that $\left(X, G_{X}\right)$ remains terminal throughout the MMP. Assumption (iii) means that the MMP terminates with a Mf.

Corollary 3.15. Let $X$ be a projective $\mathbb{Q}$-factorial variety, let $G_{X}$ be as in Setup 3.14, and let $\Delta_{1}, \ldots, \Delta_{r} \geqslant 0$ be big $\mathbb{Q}$-divisors on $X$ such that all pairs $\left(X, G_{X}+\Delta_{i}\right)$ are klt.

Then for all $\varepsilon>0$ there are ample $\mathbb{Q}$-divisors $H_{1}, \ldots, H_{r} \geqslant 0$ with $\left\|H_{i}\right\|<\varepsilon$ such that

$$
\mathcal{C}^{\prime}=\left\langle K_{X}+G_{X}, K_{X}+G_{X}+\Delta_{1}+H_{1}, \ldots, K_{X}+G_{X}+\Delta_{r}+H_{r}\right\rangle
$$

is generic.

Proof. Add enough ample divisors to span $N^{1}$ and then use Lemma 3.12 to perturb $\Delta_{1}, \ldots, \Delta_{r}$ inside a bigger cone. Since $K_{X}+G_{X} \notin \overline{\operatorname{Eff}} X$, we have $K_{X}+G_{X} \notin \operatorname{Supp} \mathfrak{R}(X, \mathcal{C})$, and hence there is no need to perturb $G_{X}$.

3.2.2 Special case: 2 -dimensional cones Suppose that $A$ is a big $\mathbb{Q}$-divisor on $X$ such that

(i) $\left(X, G_{X}+A\right)$ is klt;

(ii) $K_{X}+G_{X}+A$ is ample on $X$;

(iii) $\mathcal{C}=\left\langle K_{X}+G_{X}, K_{X}+G_{X}+A\right\rangle$ is generic.

\footnotetext{
${ }^{3} \mathrm{~A} \mathbb{Q}$-divisor $M$ is mobile if for some integer $n>0$ such that $n M$ is integral, the linear system $|n M|$ has no fixed (divisorial) part.
} 


\section{The SARkisov program for Mori fibred CAlabi-Yau Pairs}

(1) The decomposition of Supp $\Re(X, \mathcal{C})$ given by Theorem 3.8 corresponds to running a MMP for $K_{X}+G_{X}$ with scaling by $A$. This MMP exists by [BCHM10, Corollary 1.4.2]. In more detail, let

$$
1=t_{0}>t_{1}>\cdots>t_{N+1}>0
$$

be rational numbers such that $\operatorname{Supp} \mathfrak{R}(X, \mathcal{C})=\left\langle K_{X}+G_{X}+A, K_{X}+G_{X}+t_{N+1} A\right\rangle$ and the maximal cones of the decomposition correspond to the intervals $\left(t_{i}, t_{i+1}\right)$. For all $t \in\left(t_{i}, t_{i+1}\right)$, the divisor $K_{X}+G_{X}+t A$ is ample on $X_{i}=\operatorname{Proj} R\left(X, K_{X}+G_{X}+t A\right)$. Then

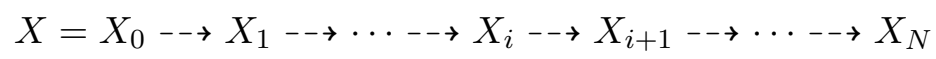

is a minimal model program for $K_{X}+G_{X}$ with scaling by $A$; that is,

(i)

$$
t_{i+1}=\inf \left\{\tau \in \mathbb{R} \mid K_{X_{i}}+G_{X_{i}}+\tau A_{i} \text { is nef }\right\},
$$

where $A_{i}$ denotes the push-forward of $A$ and $X_{i} \rightarrow X_{i+1}$ is the divisorial contraction or flip of an extremal ray $R_{i} \subset \overline{\mathrm{NE}}\left(X_{i}\right)$ with

$$
\left(K_{X_{i}}+G_{X_{i}}+t_{i+1} A_{i}\right) \cdot R_{i}=0 \quad \text { and } \quad\left(K_{X_{i}}+G_{X_{i}}\right) \cdot R_{i}<0
$$

(ii)

$$
t_{N+1}=\inf \left\{\lambda \mid K_{X}+G_{X}+\lambda A \text { is effective }\right\},
$$

where $K_{X}+G_{X}+\lambda A$ effective means that it is $\mathbb{Q}$-linearly equivalent to an effective divisor, and

$$
X_{N} \rightarrow \operatorname{Proj} R\left(X, K_{X}+G_{X}+t_{N+1} A\right)
$$

is a Mf.

(2) Genericity means that at each step there is a unique extremal ray $R_{i} \subset \overline{\mathrm{NE}}\left(X_{i}\right)$ with $\left(K_{X_{i}}+G_{X_{i}}+t_{i+1} A_{i}\right) \cdot R_{i}=0$ and $\left(K_{X_{i}}+G_{X_{i}}\right) \cdot R_{i}<0$.

(3) The genericity immediately implies the following: If $0<\varepsilon \ll 1$ is small enough, then for all ample $\mathbb{Q}$-divisors $H$ with $\|H\|<\varepsilon$, the divisor $K_{X}+G_{X}+A+H$ is ample on $X$, the cone $\mathcal{C}^{\prime}=\left\langle K_{X}+G_{X}, K_{X}+G_{X}+A^{\prime}\right\rangle$ is still generic, and the MMP for $K_{X}+G_{X}$ with scaling by $A+H$ is identical to the MMP for $K_{X}+G_{X}$ with scaling by $A$, in the sense that the sequence of steps and end product are identical.

3.2.3 Special case: 3 -dimensional cones In this subsection we prove the following special case of Theorem 3.3.

Lemma 3.16. Suppose that $\left(Y, G_{Y}\right)$ is as in Setup 3.14 and that $A$ and $A^{\prime}$ are big $\mathbb{Q}$-divisors on $Y$ such that

(i) $\left(Y, G_{Y}+A\right)$ and $\left(Y, G_{Y}+A^{\prime}\right)$ are klt;

(ii) $K_{Y}+G_{Y}+A$ and $K_{Y}+G_{Y}+A^{\prime}$ are both ample on $Y$;

(iii) $\mathcal{C}=\left\langle K_{Y}+G_{Y}, K_{Y}+G_{Y}+A\right\rangle$ and $\mathcal{C}^{\prime}=\left\langle K_{Y}+G_{Y}, K_{Y}+G_{Y}+A^{\prime}\right\rangle$ are generic;

(iv) the $M M P$ for $K_{Y}+G_{Y}$ with scaling by $A$, respectively $A^{\prime}$, ends in a $M f X / S$, respectively $X^{\prime} / S^{\prime}$.

Then the birational map $\varphi: X \rightarrow X^{\prime}$ is a composition of links $\varphi_{i}: X_{i} / S_{i} \rightarrow X_{i+1} / S_{i+1}$ of the Sarkisov program, where each map $Y \rightarrow X_{i}$ is contracting.

Proof. The proof is the argument of [HM13], which we sketch here for the reader's convenience. After a small perturbation of $A$ and $A^{\prime}$ as in Corollary 3.15 that, as stated in Section 3.2.2(3), 


\section{A. Corti And A.-S. Kaloghiros}

does not change the two MMPs or their end products, the cone $\widetilde{\mathcal{C}}=\left\langle K_{Y}+G_{Y}, K_{Y}+G_{Y}+A, K_{Y}+\right.$ $\left.G_{Y}+A^{\prime}\right\rangle$ is generic. The argument of [HM13] then shows how walking along the boundary of Supp $\widetilde{\mathcal{C}}$ corresponds to a chain of Sarkisov links from $X / S$ to $X^{\prime} / S^{\prime}$. By construction, all maps from $Y$ are contracting.

\subsection{Proof of Theorem 3.3}

Write $\chi=\chi_{N-1} \circ \cdots \circ \chi_{0}$, where each

$$
\chi_{i}: Y_{i} \rightarrow Y_{i+1}
$$

is a Mori flip, flop or inverse flip, and $Y=Y_{0}, Y^{\prime}=Y_{N}$.

For all $\mathbb{Q}$-divisors $G_{Y}$ on $Y$ denote by $G_{Y_{i}}$ the strict transform on $Y_{i}$. Choose $G_{Y}$ such that for all $i \in\{0, \ldots, N\}$

(i) $G_{Y_{i}}$ satisfies the Setup 3.14;

(ii) $\chi_{i}$ is either a $\left(K_{Y_{i}}+G_{Y_{i}}\right)$-flip or antiflip. ${ }^{4}$

One way to choose $G_{Y}$ is as follows: if $\chi_{i}$ is a flop, choose $G_{Y_{i}}$ ample, general and very small on $Y_{i}$. If $G_{Y_{i}}$ is small enough, then for all $j$ if $\chi_{j}$ was a flip or antiflip, then it still is a flip or antiflip. On the other hand, now $\chi_{i}$ is a $\left(K_{Y_{i}}+G_{Y_{i}}\right)$-flip. Some other flops may have become flips or antiflips. If there are still flops, repeat the process by adding, on $Y_{k}$ such that $\chi_{k}$ is a $\left(K_{Y_{k}}+G_{Y_{k}}\right)$-flop, a very small ample divisor to $G_{Y_{k}}$, and so on until there are no flops left.

For all $i \in\{0, \ldots, N\}$, we choose by induction on $i$ a big divisor $A_{i}$ on $Y_{i}$ such that $K_{Y_{i}}+$ $G_{Y_{i}}+A_{i}$ is ample, $\left\langle K_{Y_{i}}+G_{Y_{i}}, K_{Y_{i}}+G_{Y_{i}}+A_{i}\right\rangle$ is generic, and the MMP for $K_{Y_{i}}+G_{Y_{i}}$ with scaling by $A_{i}$ terminates with a Mf $p_{i}: X_{i} \rightarrow S_{i}$. (Note that $A_{j}$ is not the transform of $A_{i}$ on $Y_{j}$ : it is just another divisor.) At the start $p_{0}=p: X_{0}=X \rightarrow S_{0}=S$, but it will not necessarily be the case that $p_{N}=p^{\prime}$. We prove, also by induction on $i$, that for all $i$ the induced map $\varphi_{i}: X_{i--\rightarrow} X_{i+1}$ is the composition of Sarkisov links under $Y$. Finally, we prove that the induced map $X_{N} \rightarrow X^{\prime}$ is the composition of Sarkisov links under $Y$.

Suppose that for all $j<i$, we have constructed $A_{j}$. We consider two cases.

(1) If $\chi_{i-1}$ is a $\left(K_{Y_{i-1}}+G_{Y_{i-1}}\right)$-flip, choose an ample $\mathbb{Q}$-divisor $A_{i-1}^{\prime}$ on $Y_{i-1}$ such that $\left\langle K_{Y_{i-1}}+G_{Y_{i-1}}, K_{Y_{i-1}}+G_{Y_{i-1}}+A_{i-1}^{\prime}\right\rangle$ is generic and the MMP for $K_{Y_{i-1}}+G_{Y_{i-1}}$ with scaling by $A_{i-1}^{\prime}$ begins with the flip $\chi_{i-1}$. This can be accomplished as follows: If $\chi_{i-1}$ is the flip of the extremal contraction $\gamma_{i-1}: Y_{i-1} \rightarrow Z_{i-1}$, then $A_{i-1}^{\prime}=L_{i-1}+\gamma_{i-1}^{\star}\left(N_{i-1}\right)$, where $L_{i-1}$ is ample on $Y_{i-1}$ and $N_{i-1}$ is ample enough on $Z_{i-1}$. Now set

$$
A_{i}=\chi_{i-1 \star}\left(\left(t_{1}-\varepsilon\right) A_{i-1}^{\prime}\right),
$$

where $K_{Y_{i-1}}+G_{Y_{i-1}}+t_{1} A_{i-1}^{\prime}$ is $\gamma_{i-1}$-trivial and $0<\varepsilon \ll 1$. Note that $\left\langle K_{Y_{i-1}}+G_{Y_{i-1}}, K_{Y_{i-1}}+\right.$ $\left.G_{Y_{i-1}}+A_{i-1}^{\prime}\right\rangle$ generic implies $\left\langle K_{Y_{i}}+G_{Y_{i}}, K_{Y_{i}}+G_{Y_{i}}+A_{i}\right\rangle$ generic. ${ }^{5}$ We take $p_{i}: X_{i} \rightarrow S_{i}$ to be the end product of the MMP for $K_{Y_{i}}+G_{Y_{i}}$ with scaling by $A_{i}$. It follows from Lemma 3.16, applied to $Y_{i-1}$ and the divisors $A_{i-1}$ and $A_{i-1}^{\prime}$, that the induced map $\varphi_{i}: X_{i-1} \rightarrow X_{i}$ is a composition of Sarkisov links under $Y_{i-1}$ and hence, since $Y \rightarrow Y_{i-1}$ is an isomorphism in codimension 1, under $Y$.

(2) If $\chi_{i-1}$ is a $\left(K_{Y_{i-1}}+G_{Y_{i-1}}\right)$-antiflip, choose $A_{i}$ ample on $Y_{i}$ such that $\left\langle K_{Y_{i}}+G_{Y_{i}}, K_{Y_{i}}+\right.$ $\left.G_{Y_{i}}+A_{i}\right\rangle$ is generic and the MMP for $K_{Y_{i}}+G_{Y_{i}}$ with scaling by $A_{i}$ begins with the flip $\chi_{i-1}^{-1}$.

\footnotetext{
${ }^{4}$ The purpose of $G$ is to make sure that there are no flops.

${ }^{5}$ The divisor $A_{i-1}^{\prime}$ is ample, hence (moving in the linear equivalence class) $\left(Y_{i-1}, G_{Y_{i-1}}+A_{i-1}^{\prime}\right)$ is klt-in fact even terminal if we want. So since $t_{1}<1$, we even have that $\left(Y_{i}, G_{Y_{i}}+A_{i}\right)$ is klt.
} 


\section{The Sarkisov Program for Mori fibred CALABi-Yau Pairs}

We take $p_{i}: X_{i} \rightarrow S_{i}$ to be the end product of the MMP for $K_{Y_{i}}+G_{Y_{i}}$ with scaling by $A_{i}$. It follows from Lemma 3.16, applied to $Y_{i-1}$ and the divisors $A_{i-1}$ and $A_{i-1}^{\prime}=\chi_{i-1 \star}^{-1}\left(\left(t_{1}-\varepsilon\right) A_{i}\right)$, where $K_{Y_{i}}+G_{Y_{i}}+t_{1} A_{i}$ is $\chi_{i-1}^{-1}$-trivial and $0<\varepsilon \ll 1$, that the induced map $\varphi_{i-1}: X_{i-1}-\rightarrow X_{i}$ is a composition of Sarkisov links under $Y_{i-1}$ and hence, since $Y \rightarrow Y_{i-1}$ is an isomorphism in codimension 1, under $Y$.

Finally, choose $A^{\prime}$ ample on $Y^{\prime}$ such that $\left\langle K_{Y^{\prime}}+G_{Y^{\prime}}, K_{Y^{\prime}}+G_{Y^{\prime}}+A^{\prime}\right\rangle$ is generic and the MMP for $K_{Y^{\prime}}+G_{Y^{\prime}}$ with scaling by $A^{\prime}$ terminates with the Mf $p^{\prime}: X^{\prime} \rightarrow S^{\prime}$. It follows from Lemma 3.16, applied to $Y_{N}=Y^{\prime}$ and the divisors $A_{N}$ and $A^{\prime}$, that the induced map $\varphi_{N}: X_{r}-\rightarrow X^{\prime}$ is a composition of Sarkisov links under $Y^{\prime}$ and hence, since $Y \rightarrow Y^{\prime}$ is an isomorphism in codimension 1, under $Y$.

\section{Proof of Theorem 1.1}

Let $(X, D)$ and $\left(X^{\prime}, D^{\prime}\right)$ with $p: X \rightarrow S$ and $p^{\prime}: X^{\prime} \rightarrow S^{\prime}$ be (t,lc) Mf CY pairs, and let $\varphi: X-\rightarrow$ $X^{\prime}$ be a volume-preserving birational map. Theorem 1.9 gives a diagram

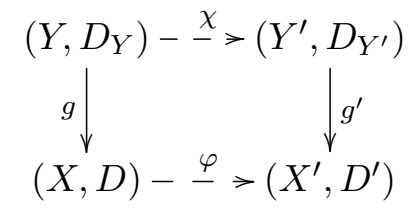

where $\left(Y, D_{Y}\right)$ and $\left(Y^{\prime}, D_{Y^{\prime}}\right)$ are $(\mathrm{t}, \mathrm{dlt}) \mathbb{Q}$-factorial CY pairs, $g$ and $g^{\prime}$ are volume preserving, and $\chi$ is a volume-preserving composition of Mori flips, flops and inverse flips. In particular, if we forget the divisors $D$, we are in the situation of Section 3.1, so that by Theorem 3.3, the map $\varphi: X \rightarrow X^{\prime}$ is the composition of Sarkisov links $\varphi_{i}: X_{i} / S_{i} \rightarrow X_{i+1} / S_{i+1}$ such that all induced maps $g_{i}: Y \rightarrow X_{i}$ are contracting. It is clear that for all $i$, the map $g_{i}$ is volume preserving, hence for $D_{i}=g_{i \star} D_{Y}$, the map $\varphi_{i}:\left(X_{i}, D_{i}\right) \rightarrow\left(X_{i+1}, D_{i+1}\right)$ also is volume preserving, and $\left(X_{i}, D_{i}\right)$ is a $(\mathrm{t}, \mathrm{lc}) \mathrm{CY}$ pair.

\section{ACKNOWLEDGEMENTS}

We thank Paolo Cascini, János Kollár and Vladimir Lazić for useful comments on a preliminary version of this paper.

\section{REFERENCES}

BCHM10 C. Birkar, P. Cascini, C.D. Hacon and J. McKernan, Existence of minimal models for varieties of log general type, J. Amer. Math. Soc. 23 (2010), no. 2, 405-468; http://dx.doi.org/10. 1090/S0894-0347-09-00649-3.

Bla13 J. Blanc, Symplectic birational transformations of the plane, Osaka J. Math. 50 (2013), no. 2, 573-590; http://projecteuclid.org/euclid.ojm/1371833501.

BM97 A. Bruno and K. Matsuki, Log Sarkisov program, Internat. J. Math. 8 (1997), no. 4, 451-49; http://dx.doi.org/10.1142/S0129167X97000238.

Cor95 A. Corti, Factoring birational maps of threefolds after Sarkisov, J. Algebraic Geom. 4 (1995), no. 2, 223-254.

Fuj11 O. Fujino, Semi-stable minimal model program for varieties with trivial canonical divisor, Proc. Japan Acad. Ser. A Math. Sci. 87 (2011), no. 3, 25-30; http://dx.doi.org/10.3792/pjaa. 


\section{A. Corti And A.-S. Kaloghiros}

87.25 .

HM13 C. D. Hacon and J. McKernan, The Sarkisov program, J. Algebraic Geom. 22 (2013), no. 2, 389-405; http://dx.doi.org/10.1090/S1056-3911-2012-00599-2.

KKL12 A.-S. Kaloghiros, A. Küronya and V. Lazić, Finite generation and geography of models, Minimal Models and Extremal Rays (S. Mukai, ed.), Adv. Stud. Pure Math. (Math. Soc. Japan, Tokyo), to appear, arXiv:1202.1164.

KM98 J. Kollár and S. Mori, Birational geometry of algebraic varieties, Cambridge Tracts in Math., vol. 134 (Cambridge Univ. Press, Cambridge, 1998); http://dx.doi.org/10.1017/ CB09780511662560.

Kol13 J. Kollár, Singularities of the minimal model program, Cambridge Tracts in Math., vol. 200 (Cambridge Univ. Press, Cambridge, 2013); http://dx.doi.org/10.1017/ CB09781139547895.

$\mathrm{Kol}^{+} 92$ J. Kollár et al., Flips and abundance for algebraic threefolds, Papers from the Second Summer Seminar on Algebraic Geometry (University of Utah, August 1991), Astérisque (1992), no. 211 (Soc. Math. France, Paris, 1992).

KSC04 J. Kollár, K.E. Smith and A. Corti, Rational and nearly rational varieties, Cambridge Stud. Adv. Math., vol. 92 (Cambridge Univ. Press, Cambridge, 2004).

KX15 J. Kollár and C. Xu, The dual complex of Calabi-Yau pairs, arXiv:1503.08320.

Usn06 A. Usnich, Symplectic automorphisms of $\mathbb{C P}^{2}$ and the Thompson group $T$, arXiv:math.AG/0611604.

Alessio Corti a.corti@imperial.ac.uk

Department of Mathematics, Imperial College London, 180 Queen's Gate, London, SW7 2AZ, United Kingdom

Anne-Sophie Kaloghiros anne-sophie.kaloghiros@brunel.ac.uk

Department of Mathematics, Brunel University, Uxbridge, Middlesex, UB8 3PH, United Kingdom 